\title{
Chemical functionalization of GaN and AIN surfaces
}

\author{
B. Baur, G. Steinhoff, and J. Hernando \\ Walter Schottky Institut, Technische Universität München, Am Coulombwall, D-85748 Garching, Germany
}

O. Purrucker and M. Tanaka

Physik-Department E22, Technische Universität München, James-Franck-Strasse, D-85748 Garching, Germany

\author{
B. Nickel \\ Department of Physics, Ludwig-Maximilians-University, Geschwister-Scholl-Platz, \\ D-80539 München, Germany
}

\author{
M. Stutzmann and M. Eickhoff ${ }^{\text {a) }}$ \\ Walter Schottky Institut, Technische Universität München, Am Coulombwall, D-85748 Garching, Germany
}

(Received 3 August 2005; accepted 26 October 2005; published online 22 December 2005)

\begin{abstract}
The covalent functionalization of GaN and AlN surfaces with organosilanes is demonstrated. Both octadecyltrimethoxysilane and aminopropyltriethoxysilane form self-assembled monolayers on hydroxylated GaN and AlN surfaces, confirmed by x-ray photoelectron spectroscopy and atomic force microscopy. The monolayer thickness on GaN was determined to $2.5 \pm 0.2 \mathrm{~nm}$ by $\mathrm{x}$-ray reflectivity. Temperature-programmed desorption measurements reveal a desorption enthalpy of $240 \mathrm{~kJ} / \mathrm{mol}$. The realization of micropatterned self-assembled monolayers and the hybridization of deoxyribonucleic acid molecules on biofunctionalized GaN surfaces are shown. (C) 2005 American Institute of Physics. [DOI: 10.1063/1.2150280]
\end{abstract}

Group III-nitrides attract increasing interest as a substrate material for bioelectronic applications. It has recently been shown that they are nontoxic, exhibit long-term chemical stability under physiological conditions, and can serve as a substrate material for living cells. ${ }^{1} \mathrm{AlGaN} / \mathrm{GaN}$ electrolyte gate field effect transistors, operated as ion-sensitive devices, show an almost Nernstian $p$ H-response of about $56 \mathrm{mV} / p \mathrm{H}^{1}{ }^{1}$ Extracellular electrical recording of action potentials with AlGaN/GaN EGFET arrays has recently been demonstrated. ${ }^{2}$

The application of these devices for electronic detection of specific biomolecular processes is a promising approach for novel biosensors based on molecular recognition, such as specific antibody detection or label-free detection of deoxyribonucleic acid (DNA) hybridization. For this purpose, the covalent attachment of specific molecules with controlled structural order and composition on group III-nitride devices is a basic requirement.

Covalent coupling of biomolecules to oxidized and hydrogen-terminated silicon surfaces has been investigated in numerous works during recent decades. ${ }^{3,4}$ Covalent grafting of self-assembled monolayers (SAMs) of organosilanes on oxidized silicon surfaces ${ }^{5-7}$ is widely used as the first step of surface functionalization with biomolecules for electronic detection of enzyme activity and DNA hybridization with Si-based field effect transistors. ${ }^{8,9}$ In contrast, the surface chemistry on AlGaN alloys has not yet been studied in great detail. Pioneering work has been carried out by Bermudez, ${ }^{10,11}$ who has analyzed the adsorption of different organic molecules, such as anilines or octanethiols on GaN surfaces, from the gas phase. In a recent publication Kang et al. $^{12}$ have reported the electrical detection of immobilized proteins on $\mathrm{AlGaN} / \mathrm{GaN}$ transistors, modified with aminopropyltriethoxysilane (APTES) molecules in a liquid phase

\footnotetext{
a) Author to whom correspondence should be addressed; electronic mail:
} eickhoff@wsi.tum.de reaction. However, the covalent immobilization of molecules was not proven.

In this letter, we report the covalent attachment of SAMs of octadecyltrimethoxysilane (ODTMS) and APTES on hydroxylated GaN and AlN surfaces. Complementary analysis by x-ray photoelectron spectroscopy (XPS), atomic force microscopy (AFM), and temperature-programmed desorption mass spectrometry proves covalent coupling of the SAMs to the surface. Immobilization of single-stranded 20-mer oligonucleotides on APTES-modified nitride surfaces and hybridization is demonstrated by fluorescence microscopy.

$\mathrm{GaN}$ and AlN layers with a thickness of $2 \mu \mathrm{m}$ were grown by metalorganic chemical vapor deposition (MOCVD) on $c$-plane sapphire substrates. AFM over a scan area of $4 \mu \mathrm{m}^{2}$ revealed a root-mean-square surface roughness of $0.2 \mathrm{~nm}$ for $\mathrm{GaN}$ and $0.3 \mathrm{~nm}$ for AlN, respectively.

The presence of hydroxyl groups on the hydrophilic surface is an essential requirement for the silanization process. $^{5-7}$ Therefore, the substrates were immersed in $\mathrm{H}_{2} \mathrm{SO}_{4}: \mathrm{H}_{2} \mathrm{O}_{2}$ (3:1) solution for $20 \mathrm{~min}$, rinsed with deionized water (18 $\mathrm{M} \Omega \mathrm{cm}$, Millipore) and dried under nitrogen flux prior to silanization. In addition to the activation of surface hydroxyl groups, this treatment leads to the formation of a thin oxide layer on GaN which has been proven by XPS. Figure 1(a) displays the evolution of the $\mathrm{O} 1 \mathrm{~s}$ core level emission of $\mathrm{GaN}$ and $\mathrm{AlN}$ measured with a $\mathrm{Mg} K \alpha$ x-ray source operated at $8 \mathrm{kV}$ and $12 \mathrm{~mA}$. For GaN, a clear intensity increase and a shift of the Ga $2 p$ core level of about $0.3 \mathrm{eV}$ to higher binding energies (not shown) is observed. In the case of AlN, the native oxide layer present on the surface is not significantly enhanced indicating that the wet chemical treatment only results in surface hydroxylation. No influence on the intensity and peak position of the $\mathrm{Al} 2 p$ emission was found. X-ray reflectivity measurements revealed an oxide thickness of $10 \pm 2 \AA(11 \pm 2 \AA)$ for the hydroxylated GaN (AIN) surface. In contrast to thermal oxidation, which leads to an increase of the surface roughness, ${ }^{13}$ AFM measure- 


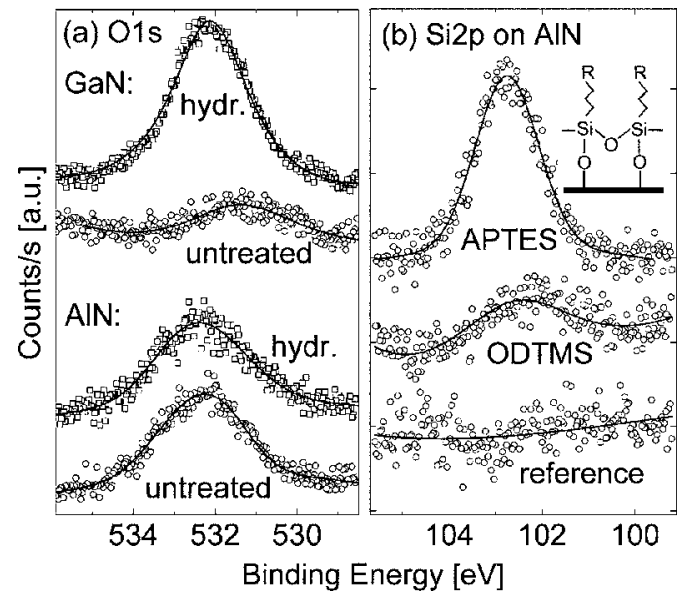

FIG. 1. (a) O $1 s$ core-level XPS-Spectra of untreated (circles) and hydroxylated (squares) AlN and GaN. For GaN, an enhanced surface oxidation is observed. (b) Si $2 p$ core-level peaks for hydroxylated as well as APTES- and ODTMS-modified AlN. The inset shows a schematic of SAM formation on the surface (R: Remaining alkyl chain for ODTMS, amino group for APTES).

ments did not reveal any influence of the wet chemical process on the surface morphology. The resulting hydrophilic properties are mirrored by a decrease of the water contact angle from $74^{\circ}\left(57^{\circ}\right)$ to $<10^{\circ}$ for $\mathrm{GaN}$ (AIN).

After hydroxylation the substrates were dried for $12 \mathrm{~h}$ in an exsiccator. The deposition of ODTMS was then performed by sonication for $60 \mathrm{~min}$ in a solution of 5\% ODTMS $(95 \%, \mathrm{ABCR})$ in toluene (puriss, Fluka) with $0.5 \%$ butylamine (99.5\%, Aldrich) as a catalyst according to Ref. 14. Sonication in toluene and methanol removed noncovalently attached molecules. After this process, GaN and AlN surfaces exhibited a hydrophobic behavior with a static water contact angle of $97^{\circ}$ and $96^{\circ}$, respectively, which remains stable over several weeks.

Deposition of APTES SAMs on GaN and AlN was performed by sonication in a solution of $20 \mathrm{mM}$ APTES $(98 \%$, Sigma) in toluene for $1.5 \mathrm{~h}$ at a temperature of $50{ }^{\circ} \mathrm{C}$. To remove excess silane aggregates, the substrates were sonicated in toluene and isopropanol and washed in acetic acid solution before they were dried under nitrogen flux.

Adsorption of silane molecules schematically shown in the inset of Fig. 1(b), was proven by XPS analysis. Figure 1(b) displays the Si $2 p$ core-level emission from hydroxylated, ODTMS- and APTES-modified AlN. For both silanization processes, a clear Si $2 p$ emission with binding energies of $102.7 \pm 0.2 \mathrm{eV}$ and $102.4 \pm 0.2 \mathrm{eV}$ is found, indicating the presence of $\mathrm{Si}-\mathrm{O}$ bonds. The significantly larger $\mathrm{Si} 2 p$ peak intensity for the APTES SAMs mirrors the different chain lengths $\left(0.85 \mathrm{~nm}\right.$ for APTES and $2.62 \mathrm{~nm}$ for ODTMS). ${ }^{15}$

Due to the spectral overlap with the Ga $3 p$ substrate emission the Si $2 p$ core-level peak is hardly detectable for silanized GaN. Here, the weaker Si $2 s$ peak was recorded and showed similar behavior as the $\mathrm{Si} 2 p$ level for AlN.

Figure 2(a) shows the $\mathrm{N} 1 s$ core-level emission of APTES-modified AlN. Adsorption of APTES is indicated by the asymmetric signal of the neutral and positively charged amino tail groups at binding energies of $399.7 \pm 0.2 \mathrm{eV}$ $\left(\mathrm{NH}_{2}\right)$ and $401.4 \pm 0.2 \mathrm{eV}\left(\mathrm{NH}_{3}^{+}\right)$, displayed in Fig. 2(b). This behavior, previously reported for silicon dioxide surfaces by Bierbaum et al., ${ }^{6}$ was also observed for APTESmodified $\mathrm{GaN}$. Quantitative analysis of the evolution of the Downloaded 27 Mar 2007 to 131.169.95.147. Redistribution subject

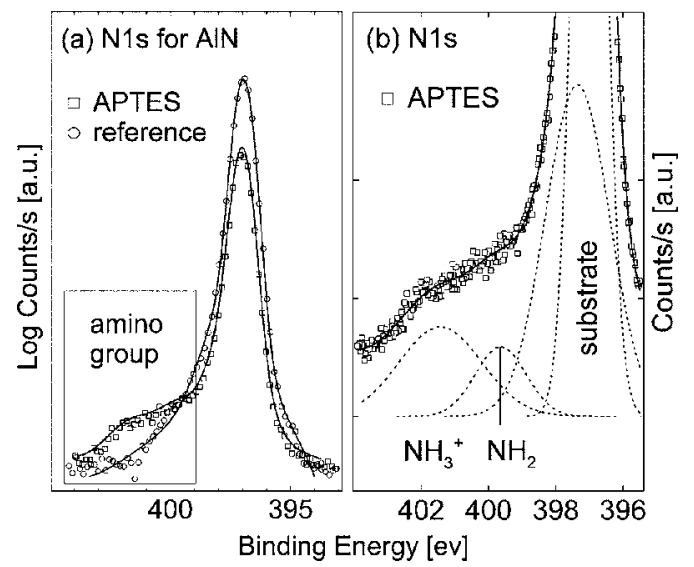

FIG. 2. (a) N $1 s$ core-level XPS spectra of the AIN surface after hydroxylation and after deposition of an APTES SAM. The shoulder indicates the presence of amino groups. (b) Magnification of (a) for APTES-modified AlN. Deconvolution (dashed lines) reveals two contributions of the amino tail group.

$\mathrm{Al} 2 p, \mathrm{Ga} 3 d, \mathrm{Ga} 2 p_{3 / 2}, \mathrm{C} 1 s$, and $\mathrm{O} 1 s$ core-level peaks (not shown) indicates an ODTMS-layer thickness of $1.7 \pm 0.4 \mathrm{~nm}$ $(1.8 \pm 0.4 \mathrm{~nm})$ for $\mathrm{GaN}(\mathrm{AlN})$.

$\mathrm{X}$-ray reflectivity measurements, carried out at the Hamburger Synchrotronstrahlungslabor HASYLAB (Germany), revealed an ODTMS SAM thickness on GaN of $2.5 \pm 0.2 \mathrm{~nm}$. The good agreement with the length of the ODTMS molecule indicates a small tilting angle of the ODTMS SAM. From the extracted electron density, we determined the area occupied by one ODTMS molecule to $20.7 \pm 0.5 \AA^{2}$, implying that approximately $50 \%$ of the surface hydroxyl groups ${ }^{16}$ are covered. Besides an inhomogeneous surface coverage the discrepancy between the two methods can originate from the presence of physisorbed surface contaminants on the XPS reference sample.

SAM deposition did not change the morphology of GaN and AlN surfaces, as shown in the AFM images of a GaN [Fig. 3(a), (b)], which display that the typical atomic terraces of two-dimensional growth are still visible after hydroxylation and after SAM deposition, indicating the absence of multilayer formation or surface agglomeration of APTES molecules.
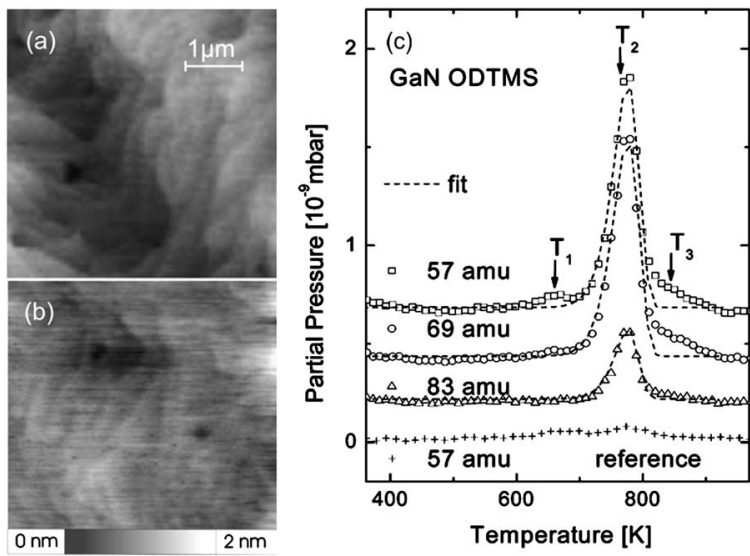

FIG. 3. AFM image of hydroxylated (a) and APTES-modified (b) GaN, showing the typical morphology due to two-dimensional growth. No silane agglomeration can be observed. (c) TPD spectra of ODTMS fragments $\mathrm{C}_{4} \mathrm{H}_{9}, \mathrm{C}_{5} \mathrm{H}_{9}$ and $\mathrm{C}_{6} \mathrm{H}_{11}$ (57 amu, $69 \mathrm{amu}$, and $83 \mathrm{amu}$ ) for ODTMSmodified GaN. Spectra are vertically shifted. 


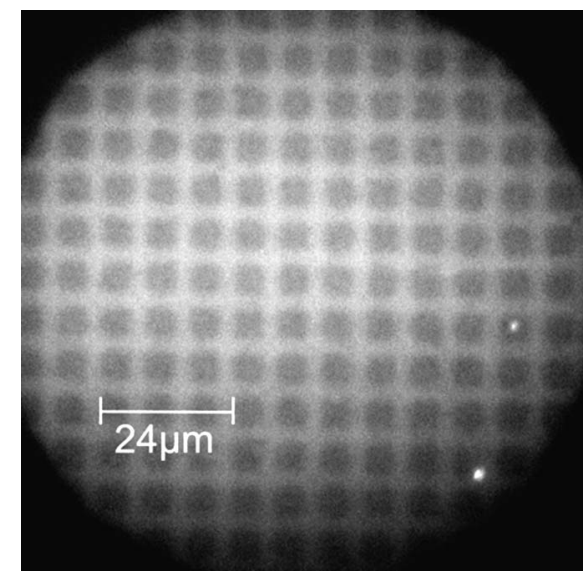

FIG. 4. Fluorescence image of alexa fluor 488-labeled single-stranded DNA after hybridization on patterned APTES-functionalized GaN. Dark regions represent areas of a degraded APTES-SAM.

Figure 3(c) shows the TPD spectra of ODTMSfunctionalized $\mathrm{GaN}$ for typical fragments of $\left(\mathrm{CH}_{2}\right)_{18}$ alkane chains, ${ }^{17}$ recorded at a heating ramp of $10^{\circ} \mathrm{C} / \mathrm{min}$. A sharp desorption peak at $T_{2}=780 \pm 20 \mathrm{~K}$ is observed. This corresponds to a desorption enthalpy of $240 \pm 20 \mathrm{~kJ} / \mathrm{mol}$, assuming a first-order desorption process for (sub)monolayer surface coverage ${ }^{18}$ which fits to the asymmetric line shape of the experimental data. The origin of the slight shoulder at $T_{3}=850 \mathrm{~K}$ is not clear yet. These results agree with Ref. 19 where degradation of ODTMS SAMs on oxidized silicon (100) surfaces was found between $740 \mathrm{~K}$ and $815 \mathrm{~K}$ and assigned to cleavage of $\mathrm{C}-\mathrm{C}$ bonds. For $\mathrm{C}_{4} \mathrm{H}_{9}$ fragment (57 amu) a minor peak at $T_{1}=650 \mathrm{~K}$ is observed for reference and ODTMS-modified surfaces. This has been previously assigned to desorption of physisorbed hydrocarbon contaminants. ${ }^{20}$ The thermal desorption of APTES SAMs exhibits the same temperature behavior, independent from the respective substrate. This indicates that cleavage of the $\mathrm{Si}-$ $\mathrm{O}-\mathrm{Ga}(\mathrm{Al})$ bond does not initiate the desorption process.

Possible applications of alkylsilane SAMs are determined by their functional group. While the hydrophobic methyl group of ODTMS can serve as a starting point for the deposition of polymer supported lipid membranes, ${ }^{21}$ the reactive amino group of APTES allows the immobilization of biomolecules. For both applications, surface micropatterning is of considerable interest.

It was shown in Ref. 14 that ultraviolet (UV) irradiation of ODTMS-functionalized silicon dioxide surfaces results in a transition from hydrophobic to hydrophilic behavior due to light-induced ablation of the silane SAM. Here, we have patterned APTES-functionalized AlN and GaN surfaces by illumination through a photomask with a $4 \mu \mathrm{m}$ square-shaped pattern using a $\mathrm{Hg}$ lamp at a power density of $15 \mathrm{~W} / \mathrm{cm}^{2}$ for 15 min. 20-mer oligonucleotides were subsequently immobilized by Schiff-Base formation.

The substrates were immersed for $1 \mathrm{~h}$ in a solution of $20 \mathrm{mM}$ glutaraldehyde (50\% in water, Sigma), which acts as the Schiff-Base linker, rinsed with water and dried under a stream of nitrogen. Afterwards, a droplet of 20-mer 5 'amino-modified oligonucleotides, diluted in phosphate buffered saline (PBS)-buffer with $p \mathrm{H}=7.0$ to a final concentration of $1 \mu \mathrm{M}$, was deposited. After immersion for $2 \mathrm{~h}$ at room temperature in humid ambiance, the substrate was washed with water and stored in PBS. Using fluorescence Downloaded 27 Mar 2007 to 131.169.95.147. Redistribution subject labeled 5'amino-3'6-FAM-modified oligonucleotides, fluorescence microscopy analysis showed that immobilization of DNA occurred only on the nonilluminated regions of the APTES-modified surface, indicating that bonding of the crosslinker to an UV-irradiated APTES molecule is inhibited. No significant decrease of fluorescence intensity was observed over several days, confirming covalent binding of DNA to the attached crosslinker.

DNA hybridization on GaN and AlN was carried out after immobilization of label-free single-stranded DNA (20mer 5' amino-modified oligonucleotide). The complementary 20-mer oligonucleotide, fluorescence labeled with 3 'alexa fluor 488, was then deposited. Hybridization was carried out for $2 \mathrm{~h}$ before washing the sample in deionized water. Figure 4 shows a fluorescence micrograph of a patterned APTESfuntionalized GaN sample after hybridization. The fluorescence labeled DNA molecules selectively attach to those surface regions where the complementary, DNA strands were immobilized.

In conclusion, we have demonstrated the functionalization of GaN and AlN surfaces by deposition of silane SAMs after wet chemical hydroxylation, results in covalently bonded silane layers. Furthermore, we have shown the immobilization of functional biomolecules on APTES-modified GaN surfaces by Schiff-Base formation.

This work was supported by the Deutsche Forschungsgemeinschaft DFG (SFB 563, Project B11) and by the European Community within the strategic research project $\mathrm{Ga}-$ Nano (Contract No. NMP4-CT-2003-505641). The HASYLAB is acknowledged for providing the necessary beamtime for this work.

${ }^{1}$ G. Steinhoff, O. Purrucker, M. Tanaka, M. Stutzmann, and M. Eickhoff, Adv. Funct. Mater. 13, 841 (2003).

${ }^{2}$ G. Steinhoff, B. Baur, G. Wrobel, S. Ingebrandt, A. Offenhäusser, A. Dadgar, A. Krost, M. Stutzmann, and M. Eickhoff, Appl. Phys. Lett. 86, 033901 (2005).

${ }^{3}$ J. P. Cloarec, J. R. Martin, C. Polychronakos, I. Lawrence, M. F. Lawrence, and E. Souteyrand, Sens. Actuators B 58, 394 (1999).

${ }^{4}$ C. Dahmen, A. Janotta, D. Dimova-Malinovska, S. Marx, B. Jeschke, B. Nies, H. Kessler, and M. Stutzmann, Thin Solid Films 427, 201 (2003).

${ }^{5}$ J. Sagiv, J. Am. Chem. Soc. 102, 92 (1980).

${ }^{6}$ K. Bierbaum, M. Kinzler, C. Wöll, M. Grunze, G. Hähner, S. Heid, and F. Effenberger, Langmuir 11, 512 (1995).

${ }^{7}$ A. N. Parikh, D. L. Allara, I. B. Azouz, and F. Rondelez, J. Phys. Chem. 98, 7577 (1994)

${ }^{8}$ E. Souteyrand, J. P. Cloarec, J. R. Martin, C. Wilson, I. Lawrence, S. Mikkelsen, and M. F. Lawrence, J. Phys. Chem. B 101, 2980 (1997).

${ }^{9}$ F. Uslu, S. Ingebrandt, D. Mayer, S. Böcker-Meffert, M. Odenthal, and A. Offenhäusser, Biosens. Bioelectron. 19, 1723 (2004).

${ }^{10}$ V. M. Bermudez, Surf. Sci. 499, 109 (2002).

${ }^{11}$ V. M. Bermudez, Langmuir 19, 6813 (2003).

${ }^{12}$ B. S. Kang, F. Ren, L. Wang, C. Lofton, W. W. Tan, S. J. Pearton, A. Dabiran, A. Osinsky, and P. P. Chow, Appl. Phys. Lett. 87, 023508 (2005).

${ }^{13}$ S. D. Wolter, J. M. DeLucca, S. E. Mohney, R. S. Kern, and C. P. Kuo, Thin Solid Films 371, 153 (2000).

${ }^{14}$ J. F. Mooney, A. J. Hunt, J. R. McIntosh, C. A. Liberko, D. M. Walba, and C. T. Rogers, Proc. Natl. Acad. Sci. U.S.A. 93, 12287 (1996).

${ }^{15}$ D. L. Allara, A. N. Parik, and F. Rondelez, Langmuir 11, 2357 (1995).

${ }^{16}$ M. Bayer, C. Uhl, and P. Vogl, J. Appl. Phys. 97, 033703 (2005).

${ }^{17}$ NIST Standard Reference Database, Vol. 69, 2005 〈http:// www.webbook.nist.gov/chemistry

${ }^{18}$ P. A. Redhead, Vacuum 12, 203 (1962).

${ }^{19}$ G. J. Kluth, M. M. Sung, and R. Maboudian, Langmuir 13, 3775 (1997).

${ }^{20}$ O. Ambacher, M. S. Brandt, R. Dimitrov, T. Metzger, M. Stutzmann, R.

A. Fischer, A. Miehr, A. Bergmeier, and G. Dollinger, J. Vac. Sci. Technol. B 14, 3532 (1996).

${ }^{21}$ E. Sackmann, Science 271, 43 (1996). 